\title{
Pruritus in renal transplant recipients: Current state of knowledge
}

\author{
Piotr K. Krajewski ${ }^{1, B-D}$, Magdalena Krajewska ${ }^{2, A, E}$, Jacek C. Szepietowski, ${ }^{1, A, E, F}$ \\ ${ }^{1}$ Department of Dermatology, Venereology and Allergology, Wroclaw Medical University, Poland \\ 2 Department of Nephrology and Transplantation Medicine, Wroclaw Medical University, Poland \\ A - research concept and design; $\mathrm{B}$ - collection and/or assembly of data; $\mathrm{C}$ - data analysis and interpretation; \\ $D$ - writing the article; $E$ - critical revision of the article; $F$ - final approval of the article
}

\section{Address for correspondence \\ Piotr K. Krajewski}

E-mail: pkrajewski@icloud.com

Funding sources

None declared

Conflict of interest

None declared

Received on February 23, 2020

Reviewed on February 29, 2020

Accepted on May 8, 2020

Published online on June 23, 2020

\begin{abstract}
Chronic itch (Cl) is the most common symptom in dermatology. It is considered a great burden with a large impact on patients' quality of life, interfering with everyday activities. During the course of chronic kidney disease (CKD) many patients develop end-stage renal disease (ESRD)-associated CI (ESRDCI). Its pathogenesis is yet to be fully explained, but multiple mechanisms have been described, including, among others, dysregulation of calcium, phosphorus, parathyroid hormone and vitamin D axis, microinflammation, and accumulation of uremic toxins. The treatment consists of both topical and systemic therapy, but unfortunately it is usually difficult and unsatisfactory. Renal transplantation (KTX) as the best renal replacement therapy improves considerably the patient's life quality and decreases the mortality rate. However, its influence on CI has not been well described. In this review, we presented the available literature on the influence of KTx on ESRDCI and demonstrated the possible ways in which transplanted kidney could help patients suffering from Cl.
\end{abstract}

Key words: kidney transplantation, pruritus, uremia

Cite as

Krajewski PK, Krajewska M, Szepietowski JC. Pruritus in renal transplant recipients: Current state of knowledge. Adv Clin Exp Med. 2020;29(6):769-772. doi:10.17219/acem/122174

DOI

10.17219/acem/122174

\section{Copyright}

Copyright by Author(s)

This is an article distributed under the terms of the

Creative Commons Attribution 3.0 Unported (CC BY 3.0)

(https://creativecommons.org/licenses/by/3.0/) 


\section{Introduction}

Chronic kidney disease (CKD) is a global health problem affecting $13.4 \%$ of the world population (stages $1-5$ ), while $10.6 \%$ of people suffer from advanced stage of the disease (stages 3-5). ${ }^{1}$ This disease is defined as a chronic (more than 3 months) decrease in renal function or abnormal renal structure. For the diagnosis, at least 1 of the following criteria must be met: glomerular filtration rate (GFR) $<60 \mathrm{~mL} / \mathrm{min} / 1.73 \mathrm{~m}^{2}$, markers of kidney damage (albuminuria, abnormal urine sedimentation rate, tubular disorders), histologic and radiologic abnormalities, or a history of renal transplantation (KTx). It can be classified into one of 5 stages on the basis of GFR. ${ }^{2}$ The loss of kidney function leads to frequent hospitalization, lower quality of life and higher morbidity rate. Chronic kidney disease patients often suffer from higher cardiovascular risk and develop a series of complications such as hyperparathyroidism and bone disorders, anemia, and dyslipidemias. ${ }^{3}$ Another troublesome symptom for patients with advanced stages of CKD is end-stage renal disease-associated chronic itch (ESRDCI), also known as a uremic itch. It was first described in $1932^{4}$ and till today remains an important problem for both patients and the doctors; it decreases patients' quality of life and poses a significant therapeutic challenge. Although numerous therapies have been described until now, ${ }^{5}$ there are still patients suffering from persistent itch. Generally, KTx is the most effective treatment for kidney failure. However, there is still very little known about the influence of KTx on the uremic itch. Moreover, our clinical experience suggests that at least some of KTx recipients may complain of chronic itch (CI). Therefore, we decided to conduct a literature review on the topic of itch in the patients after KTx.

\section{Methods}

A literature search according to PRISMA guidelines within the Medline and SCOPUS databases was conducted in March 2020 for papers discussing the problem of itch in KTx patients without setting time limits, using the terms 'pruritus' and 'itch' in conjunction with 'kidney transplantation' or 'renal transplantation'. The search was limited to English, Polish, Spanish, and German literature. Articles that did not address the topics were excluded, and the full text of the remaining articles was subsequently reviewed. Finally, 16 articles were analyzed, from which 8 papers were included in the study (Fig. 1).

\section{Results}

The recent study by Schricker et al. shows that $12 \%$ of the renal transplant recipients (RTR) are diagnosed with CI. ${ }^{6}$ The authors reported that the prevalence of itch in KTx patients is lower than in the hemodialyzed (HD) ones $(12 \%$ and $35 \%$, respectively). The mean pruritus score assessed with visual analog scale (VAS) was 3.2 points, indicating mild itch. Moreover, there was a moderate correlation $(r=0.3 ; p=0.018)$ established between the intensity of CI and the transplant function. In the study by Altmeyer et al., 49 patients after KTx were examined and skin biopsies were taken from them. ${ }^{7}$ The authors observed that all the dermatological skin disorders associated with endstage renal disease (ESRD) (among them xerosis and generalized itch) disappeared completely after the successful transplantation. The histopathologic examination showed a normalization of uremic altered skin, including xerosis.

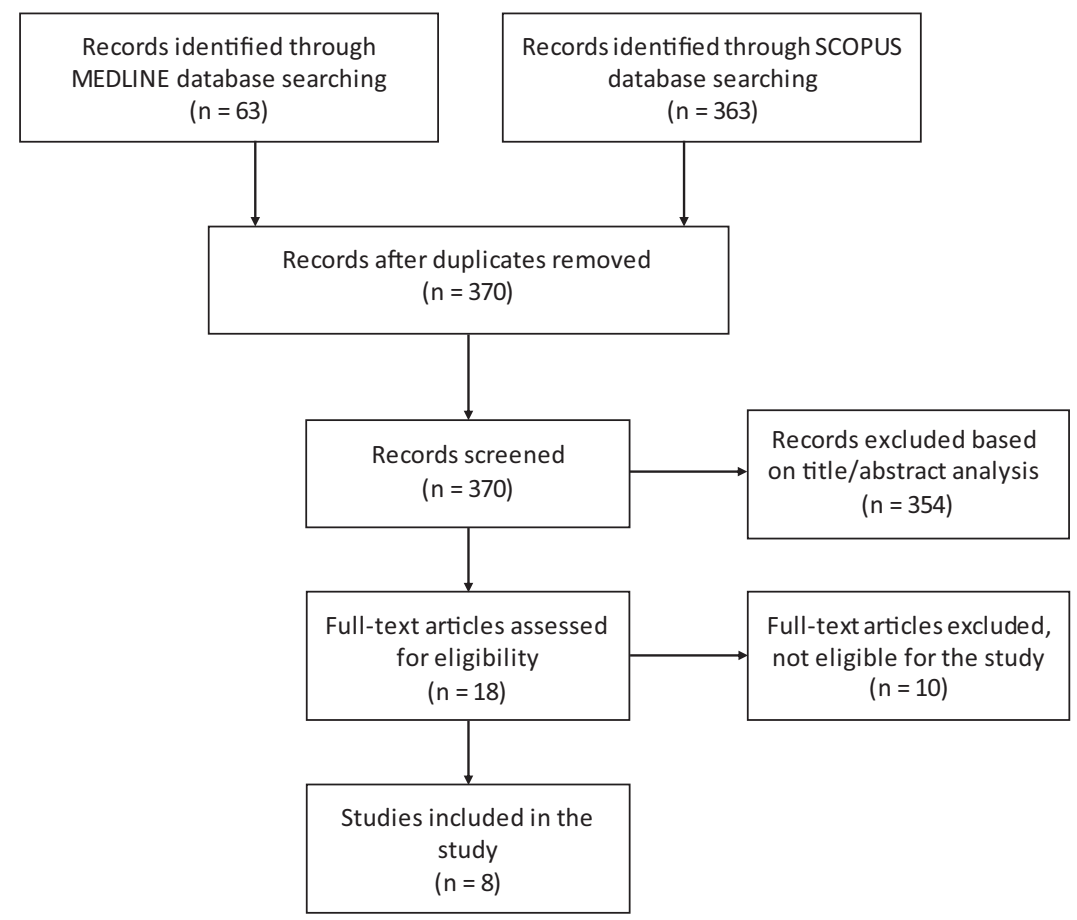

Fig. 1. Literature search procedure 
However, the alteration of connective tissue, like actinic elastosis, persisted after the transplantation. Panuccio et al. compared the prevalence of itch and its influence on the quality of life between $133 \mathrm{KTx}$ patients, $29 \mathrm{HD}$ patients and 62 healthy subjects. ${ }^{8}$ There was a significant difference $(\mathrm{p}<0.001)$ found in the itch incidence between HD and post-KTx patients (62\% and 32\%, respectively). Although the prevalence of the pruritus was lower after $\mathrm{KTx}$, it was still higher than in the healthy subjects $(32 \%$ and $11 \%$, respectively). The authors also emphasized that the itch after KTx was associated with social, emotional and working limitation ( $\mathrm{p}<0.05)$. Moloney et al. in their study reported that among 173 RTR, 43 people suffered itching (24.9\%). ${ }^{9}$ Moreover, in $57 \%$ of them, the itch had a large impact on their lives ( $>10$ points in Dermatology Life Quality Index). In the study by Amro et al., the authors analyzed 110 patients who had received a KTx and reported a statistical and clinical decrease in the negative influence on life quality by itching $(\mathrm{p}=0.001) .{ }^{10}$ Also, Avermaete et al. in their report showed a difference in itch prevalence between HD and transplant patients (48-73\% vs $2 \%) .{ }^{11}$ Furthermore, Tăranu et al. in their study reported a 5.3\% prevalence of itchiness; however, the authors associated it with immunotherapy. ${ }^{12}$ Yates et al. described a patient with generalized itchiness because of an atypical presentation of chronic scabies infestation. ${ }^{13}$ However, after successful treatment with permethrin, the itch disappeared.

\section{Discussion}

End-stage renal disease-associated chronic itch, also known as uremic pruritus or uremic itch, is a common symptom in CKD patients. It affects $35 \%$ of patients treated with HD. ${ }^{14-16}$ The sensation is usually localized, but affects whole body in almost $1 / 4$ of subjects. ${ }^{17}$ Patients suffering from uremic itch often report decreased quality of life and poor sleep quality, more frequently develop depression and their relationship satisfaction is significantly lower than in asymptomatic individuals. ${ }^{15,18,19}$ The pathogenesis of ESRDCI has not been fully explained yet and various factors are reported to play an important role in the pathogenesis of pruritus. Among them authors mention high serum levels of parathormone, urea and creatinine, xerosis, microinflammation, dysregulation of opioid receptors, and neuropathy. ${ }^{20-24}$ Due to the complex pathomechanisms and associated comorbidities, the management of uremic itch remains a therapeutic challenge. It is reported that peritoneal dialysis may be beneficial and, if not possible, adjustment of HD parameters (e.g., blood flow) may reduce itchiness. ${ }^{25}$ The treatment consists of both topical and systemic therapy. At the beginning, mostly moisturizing agents and emollients are used, but the treatment often ends with antidepressants and opioids. ${ }^{5}$

Kidney transplant is the treatment of choice in ESRD. In comparison to HD, KTx significantly improves quality of life and reduces the risk of mortality. ${ }^{26}$ Moreover, it has been proven that KTx has a big influence on ESRDCI risk factors. Firstly, the HD, in comparison to properly functioning kidney, does not effectively remove middlesize and protein-bound toxins, ${ }^{27}$ which could play a role in pruritus pathogenesis. ${ }^{28}$ Furthermore, during dialysis the interaction of peripheral blood lymphocytes with dialytic membrane increases production and release of proinflammatory cytokines, ${ }^{29}$ which ceases to be a problem after KTx. Authors also mention equilibration of calcium, phosphate, PTH, and vitamin D levels in KTx recipients. ${ }^{30}$ Furthermore, some authors reported that KTx improves neurological complication of renal failure such as uremic polyneuritis and autonomic neuropathy. ${ }^{31-33}$ Besides the existence of many reports on KTx influence on the ESRDCI risk factors, there is still very little known about pruritus in KTx recipients.

The available literature focused on this topic is very limited and consists of 7 articles. All of them state that KTx leads to the reduction of itch incidence. In the newest study by Schricker et al., the incidence of CI after KTx was $13 \%$ lower than in the HD group. ${ }^{6}$ Moreover, the transplant function was correlated with the intensity of CI. In the study by Altmeyer et al., all patients noticed the total disappearance of uremic itch. ${ }^{7}$ Although the results are promising, the report has its limitations. It was conducted on a small group of patients (49 people) and only generalized pruritus was considered. Panuccio et al. in their study compared the prevalence of itch between RTR, HD patients and healthy subjects. ${ }^{8}$ There was a statistically significant difference between the itch in KTx patients and those treated with HD (32\% and 62\%, respectively, $\mathrm{p}<0.001)$. Furthermore, the retrospectively assessed pre-transplant itch prevalence in RTR was similar to the one found in HD patients. While the post-transplant decrease in uremic itch was noted, the prevalence was still almost 3 times higher than in the general population. Moreover, no statistically significant association with well-known uremic itch risk factors was established. Nevertheless, uremic itch in the KTx patients was strongly associated $(\mathrm{p}<0.05)$ with a decrease in the quality of life, suggesting that the symptom is an important psychological burden. In a paper by Amro et al., the authors reported a statistically and clinically significant decrease in the influence of pruritus on the quality of life ( $\mathrm{p}=0.001$ and Cohen's $\mathrm{d}=0.8) .{ }^{10}$ The improvement was visible both for patients extremely bothered and moderately bothered by itch. Having this in consideration, one may conclude that KTx may have decreased itch intensity in those subjects. Additionally, Avermaete et al. reported the difference in the incidence of pruritus between RTR and the HD patients. ${ }^{11}$ Moreover, Tăranu et al. in their study reported that only $5.3 \%$ of the patients after KTx had pruritus and suggested that the observed itch was associated with immunosuppressive therapy. ${ }^{12}$

It is important to emphasize that the itch in KTx patients could be of a different origin than the uremic pruritus. 
The available reports stated that up to $88 \%$ of RTR suffer from infection ${ }^{34}$ and up to $58.4 \%$ can develop drugrelated skin manifestation, ${ }^{35}$ while in some subjects new itchy dermatoses appear (e.g., lichen planus, psoriasis and seborrheic dermatitis). ${ }^{11}$ Moreover, the possibility of persistent or tertiary hyperparathyroidism exists, which could increase pruritus. ${ }^{36}$

\section{Conclusions}

The literature on uremic itch after KTx is very limited. Although the available reports present the beneficial effect of KTx, there is still more to be discovered. Therefore, future studies on larger populations are crucial to fully evaluate the actual effect of KTx on uremic itch.

\section{ORCID iDs}

Piotr Krajewski (1) https://orcid.org/0000-0003-4722-8531

Magdalena Krajewska (1) https://orcid.org/0000-0002-2632-2409

Jacek Szepietowski 으 https://orcid.org/0000-0003-0766-6342

\section{References}

1. Hill NR, Fatoba ST, Oke JL, et al. Global prevalence of chronic kidney disease: A systematic review and meta-analysis. PLoS One. 2016;11(7): e0158765.

2. Szczeklik A, Gajewski P. Interna Szczeklika 2018. Kraków, Poland: Medycyna Praktyczna; 2018.

3. Thomas R, Kanso A, Sedor JR. Chronic kidney disease and its complications. Prim Care. 2008;35(2):329-344,vii.

4. Chargin $\mathrm{L}$, Keil $\mathrm{H}$. Skin diseases in nonsurgical renal disease. AMA Arch Derm. 1932;26(2):314-335.

5. Reszke R, Szepietowski JC. End-stage renal disease chronic itch and its management. Dermatol Clin. 2018;36(3):277-292.

6. Schricker S, Weisshaar E, Kupfer J, Mettang T. Prevalence of pruritus in a single cohort of long-term kidney transplant recipients. Acta Derm Venereol. 2020;100(4):adv00066.

7. Altmeyer P, Kachel HG, Schafer G, Fassbinder W. Normalization of uremic skin changes following kidney transplantation [in German]. Hautarzt. 1986;37(4):217-221.

8. Panuccio V, Tripepi R, Bellantoni M, et al. Pruritus and quality of life in renal transplant patients. Clin Transplant. 2017;31(3). doi:10.1111/ ctr.12893

9. Moloney FJ, Keane S, O'Kelly P, Conlon PJ, Murphy GM. The impact of skin disease following renal transplantation on quality of life. Br J Dermatol. 2005;153(3):574-578.

10. Amro A, Waldum-Grevbo B, von der Lippe N, Brekke FB, Miaskowski C, Os I. Symptom clusters from dialysis to renal transplantation: A fiveyear longitudinal study. J Pain Symptom Manage. 2016;51(3):512-519.

11. Avermaete A, Altmeyer P, Bacharach-Buhles M. Skin changes and tumours after renal transplantation. Nephron. 2002;91(2):188-194, discussion 194-186.

12. Tăranu T, Covic A, Buhaescu I, Taranu T. Drug-induced dermatological pathology in renal transplantation patients [in Romanian]. Rev Med Chir Soc Med Nat lasi. 2005;109(1):36-39.

13. Yates JE, Bleyer AJ, Yosipovitch G, Sangueza OP, Murea M. Enigmatic pruritus in a kidney transplant patient. Clin Kidney J. 2013;6(2):194-198.

14. Murtagh FE, Addington-Hall JM, Edmonds PM, et al. Symptoms in advanced renal disease: A cross-sectional survey of symptom prevalence in stage 5 chronic kidney disease managed without dialysis. J Palliat Med. 2007;10(6):1266-1276.
15. Pisoni RL, Wikstrom B, Elder SJ, et al. Pruritus in haemodialysis patients: International results from the Dialysis Outcomes and Practice Patterns Study (DOPPS). Nephrol Dial Transplant. 2006;21(12):3495-3505.

16. Hayani K, Weiss M, Weisshaar E. Clinical findings and provision of care in haemodialysis patients with chronic itch: New results from the German Epidemiological Haemodialysis Itch Study. Acta Derm Venereol. 2016;96(3):361-366.

17. Welz-Kubiak K, Reszke R, Szepietowski JC. Pruritus as a sign of systemic disease. Clin Dermatol. 2019;37(6):644-656.

18. Susel J, Batycka-Baran A, Reich A, Szepietowski JC. Uraemic pruritus markedly affects the quality of life and depressive symptoms in haemodialysis patients with end-stage renal disease. Acta Derm Venereol. 2014;94(3):276-281.

19. Ibrahim MK, Elshahid AR, El Baz TZ, Elazab RM, Elhoseiny SA, Elsaie ML. Impact of uraemic pruritus on quality of life among end stage renal disease patients on dialysis. J Clin Diagn Res. 2016;10(3):WC01-05.

20. Kaysen GA. The microinflammatory state in uremia: Causes and potential consequences. J Am Soc Nephrol. 2001;12(7):1549-1557.

21. Morton CA, Lafferty M, Hau C, Henderson I, Jones M, Lowe JG. Pruritus and skin hydration during dialysis. Nephrol Dial Transplant. 1996; 11(10):2031-2036.

22. Kurban MS, Boueiz A, Kibbi AG. Cutaneous manifestations of chronic kidney disease. Clin Dermatol. 2008;26(3):255-264.

23. Makhlough A, Emadi N, Sedighi O, Khademloo M, Bicmohamadi AR. Relationship between serum intact parathyroid hormone and pruritus in hemodialysis patients. Iran J Kidney Dis. 2013;7(1):42-46.

24. Wikstrom B, Gellert R, Ladefoged SD, et al. Kappa-opioid system in uremic pruritus: Multicenter, randomized, double-blind, placebocontrolled clinical studies. J Am Soc Nephrol. 2005;16(12):3742-3747.

25. Aliasgharpour M, Zabolypour S, Asadinoghabi A, Haghani H, Lesanpezeshki $M$. The effect of increasing blood flow rate on severity of uremic pruritus in hemodialysis patients: A single clinical trial. J Natl Med Assoc. 2018;110(3):270-275.

26. Abecassis M, Bartlett ST, Collins AJ, et al. Kidney transplantation as primary therapy for end-stage renal disease: A National Kidney Foundation/Kidney Disease Outcomes Quality Initiative (NKF/KDOQITM) conference. Clin J Am Soc Nephrol. 2008;3(2):471-480.

27. Wolley MJ, Hutchison CA. Large uremic toxins: An unsolved problem in end-stage kidney disease. Nephrol Dial Transplant. 2018; 33(Suppl 3):iii6-iii11.

28. Cupisti A, Piccoli GB, Gallieni M. Charcoal for the management of pruritus and uremic toxins in patients with chronic kidney disease. Curr Opin Nephrol Hypertens. 2020;29(1):71-79.

29. Zaza G, Pontrelli P, Pertosa G, et al. Dialysis-related systemic microinflammation is associated with specific genomic patterns. Nephrol Dial Transplant. 2008;23(5):1673-1681.

30. Bouquegneau A, Salam S, Delanaye P, Eastell R, Khwaja A. Bone disease after kidney transplantation. Clin J Am Soc Nephrol. 2016;11(7): 1282-1296.

31. Bolton CF, Baltzan MA, Baltzan RB. Effects of renal transplantation on uremic neuropathy: A clinical and electrophysiologic study. NEngl J Med. 1971;284(21):1170-1175.

32. Agarwal A, Anand IS, Sakhuja V, Chugh KS. Effect of dialysis and renal transplantation on autonomic dysfunction in chronic renal failure. Kidney Int. 1991;40(3):489-495.

33. Whitaker $\mathrm{RH}$, Hamilton D. Effect of transplantation on non-renal effects of renal failure. BrMed J (Clin Res Ed). 1982;284(6311):221-222.

34. Oh CC, Lee HY, Tan BK, Assam PN, Kee TYS, Pang SM. Dermatological conditions seen in renal transplant recipients in a Singapore tertiary hospital. Singapore Med J. 2018;59(10):519-523.

35. Alper S, Kilinc I, Duman S, et al. Skin diseases in Turkish renal transplant recipients. Int J Dermatol. 2005;44(11):939-941.

36. Yamamoto T, Tominaga $Y$, Okada M, et al. Characteristics of persistent hyperparathyroidism after renal transplantation. World J Surg. 2016;40(3):600-606. 